\title{
Relationship between the Local Structure of Orientation Map and the Strength of Orientation Tuning of Neurons in Monkey V1: A 2-Photon Calcium Imaging Study
}

\author{
Koji Ikezoe, ${ }^{1,2}$ Yoshiya Mori, ${ }^{1}$ Kazuo Kitamura, ${ }^{3,4}$ Hiroshi Tamura, ${ }^{1,2,3}$ and Ichiro Fujita ${ }^{1,2,3}$ \\ ${ }^{1}$ Laboratory for Cognitive Neuroscience, Graduate School of Frontier Biosciences, Osaka University, Toyonaka, Osaka, 560-8531, Japan, ${ }^{2} \mathrm{Center}$ for \\ Information and Neural Networks (CiNet), Osaka University and National Institute of Information and Communications Technology, Suita, Osaka, 565- \\ 0871, Japan, ${ }^{3}$ Core Research for Evolutional Science and Technology (CREST), Japan Science and Technology Agency, Kawaguchi, Saitama, 332-0012, \\ Japan, ${ }^{4}$ Department of Neurophysiology, Graduate School of Medicine, University of Tokyo, Bunkyo, Tokyo, 113-0033, Japan
}

A majority of neurons in the monkey primary visual cortex (V1) are tuned to stimulus orientations. Preferred orientations and tuning strengths vary among V1 neurons. The preferred orientation of neurons gradually changes across the cortex with occasional failures of this organization. How V1 neurons are arranged by the strength of orientation tuning and whether neuronal arrangement for tuning strength relates to orientation preference maps remains controversial. In this study, we performed in vivo two-photon calcium imaging in macaque $\mathrm{V} 1$ to examine the local spatial organization of orientation tuning at the level of single cells. We recorded fluorescence signals from individual neurons loaded with a calcium-sensitive dye in layer 2 and the uppermost tier of layer 3. The strength of orientation tuning was shared by nearby neurons, and changed across the cortex. The neurons with similar tuning strength were distributed across at least the entire thickness of layer 2. The tuning strength was weaker in regions where neurons exhibited heterogeneous preferred orientations, as compared with regions where neurons shared similar orientation preferences. Nearby direction-selective neurons often shared their preferred directions, although only a few neurons were direction selective in the layers examined. Thus, the orientation tuning strength of V1 neurons is partially predictable from the local structure of orientation map. The weaker orientation tuning we found in regions with heterogeneous orientation preferences suggests that orientation-independent interactions among local populations of $\mathrm{V} 1$ neurons play a critical role in determining their orientation tuning.

\section{Introduction}

Many neurons in the monkey striate cortex (V1) respond to a particular range of orientation of bars and gratings (Hubel and Wiesel, 1968). Preferred orientation and the strength of tuning differ among neurons (Schiller et al., 1976; De Valois et al., 1982; Ringach et al., 2002). Neurons preferring similar orientation are arrayed vertically across the cortical layers to form orientation columns (Hubel and Wiesel, 1974). In the tangential direction of the cortical surface, orientation columns smoothly vary in their preferred orientation with occasional distinct points (pinwheel centers; Blasdel and Salama, 1986; Blasdel, 1992; Nauhaus et al., 2012b) and linear fractures (Blasdel and Salama, 1986), across

Received May 24, 2013; revised Sept. 10, 2013; accepted Sept. 16, 2013.

Author contributions: K.I. and I.F. designed research; K.I., Y.M., and K.K. performed research; K.I. analyzed data; K.I., H.T., and I.F. wrote the paper.

This study was supported by grants from the Japan Science and Technology Agency (Core Research of Evolutional Science Technology); the National Institute of Information and Communication Technology (CiNet); and the Ministry of Education, Culture, Science, and Technology (23135521, 23135522, 23240047, 25135722, and 25830012). We thank Shinji Nishimoto and Kota Sasaki for helpful comments on this manuscript.

The authors declare no competing financial interests.

Correspondence should be addressed to Ichiro Fujita, Laboratory for Cognitive Neuroscience, Graduate School of Frontier Biosciences, Osaka University, 1-4 Yamadaoka, Suita, Osaka, 565-0871, Japan. E-mail: fujita@fbs.osaka-u.ac.jp.

DOI:10.1523/JNEUROSCI.2209-13.2013

Copyright $\odot 2013$ the authors $\quad 0270-6474 / 13 / 3316818-10 \$ 15.00 / 0$ which the orientation preference of columns changes in a discontinuous manner.

The relationship between the neuronal positions and the orientation tuning strength, however, is poorly understood. For example, it is controversial whether orientation tuning strength of neurons in cytochrome oxidase (CO)-abundant blobs differ from those in CO-poor interblob regions (Livingstone and Hubel, 1984; Ts'o and Gilbert, 1988; Lennie et al., 1990; Leventhal et al., 1995; O'Keefe et al., 1998; Lu and Roe, 2008; Economides et al., 2011). Furthermore, it is controversial whether orientation tuning strength of neurons is related to local heterogeneity of preferred orientations around the neurons (Bartfeld and Grinvald, 1992; Maldonado and Gray, 1996; Maldonado et al., 1997; Hetherington and Swindale, 1999; Schummers et al., 2002; Mariño et al., 2005; Ohki et al., 2006; Nauhaus et al., 2008).

To examine these relationships, it is crucial to determine the orientation preference and tuning strength of individual neurons and their exact spatial locations in local cortical regions. If activities of nearby neurons are averaged because of spike sorting error in single-unit recording or insufficient spatial resolution of intrinsic signal optical imaging, heterogeneity of preferred orientations in a local region is not correctly characterized. Moreover, in regions where preferred orientations of neurons are heterogeneous, the orientation tuning of the averaged activity across a population would become weaker than that of individual neurons. It is 
A

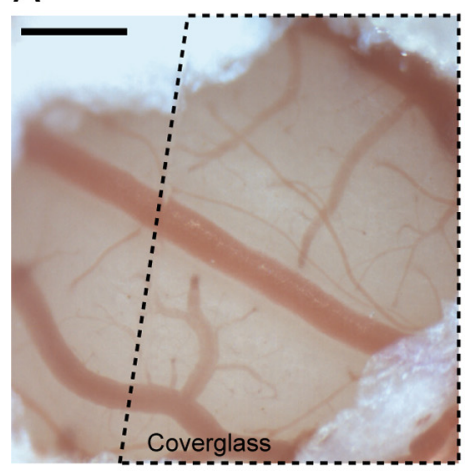

B

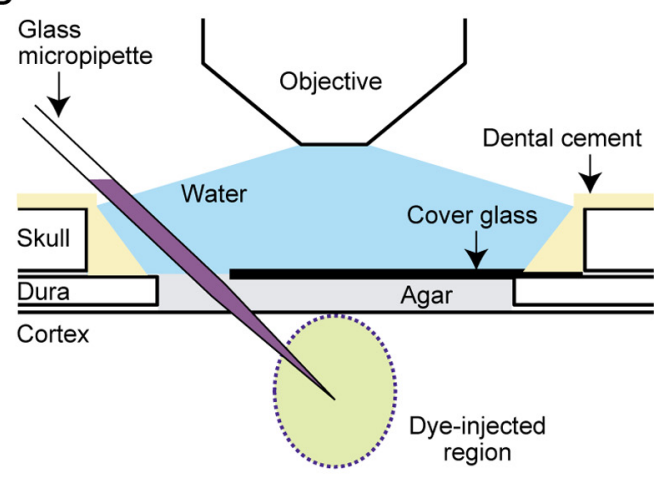

C
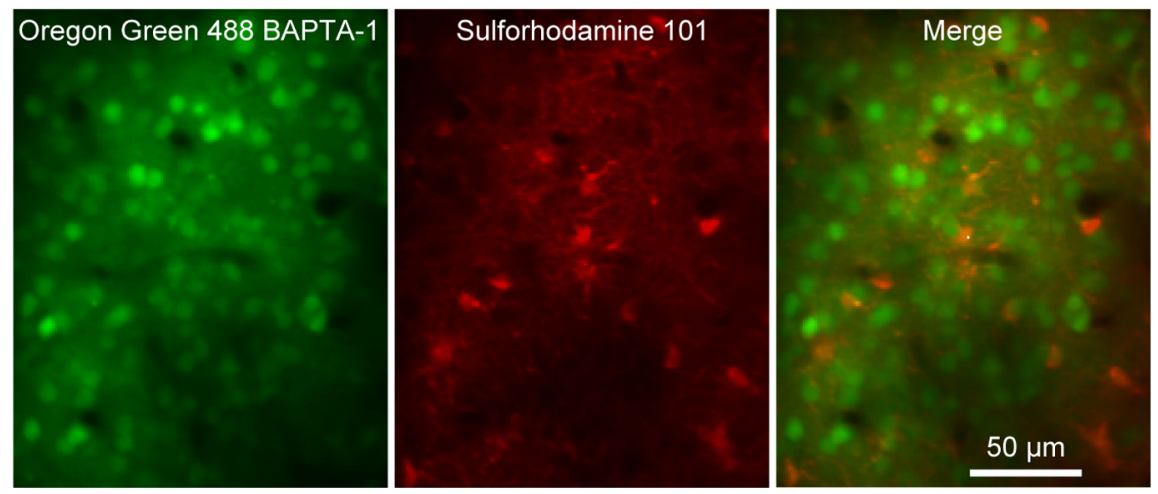

Figure 1. In vivo two-photon calcium imaging in monkey V1. $A$, An exposed cortex viewed through a hole $(\sim 1.5 \mathrm{~mm}$ in diameter) in the dura. Dotted line indicates the area covered by a microscope coverglass. Scale bar, $0.3 \mathrm{~mm}$. $\boldsymbol{B}$, Schematic illustration of the arrangement of dye injection, stabilization of the cortical surface, and imaging of calcium responses. $\boldsymbol{C}$, Structural images of a recording region. Left, Neurons and astrocytes visualized with Oregon Green 488 BAPTA-1 AM (0GB-1). Center, Astrocytes stained with sulforhodamine 101 (SR101). Right, A merged image of the left and center images.

unclear whether the weak orientation tuning results from averaging of activities across sharply tuned neurons with various preferred orientations or from clustering of weakly tuned neurons.

The recent development of in vivo two-photon calcium imaging techniques (Stosiek et al., 2003; Ohki et al., 2005) allows us to address this issue. Here, we applied this technique to the macaque V1 and investigated the relationship between the local structure of orientation map and the strength of orientation tuning of neurons. We found that nearby neurons in layers 2 and 3 were similar in both their preferred orientation and tuning strength. Orientation tuning was weak in regions with varied preferred orientations among nearby neurons, such as orientation fractures, whereas orientation tuning strength varied in regions consisting of neurons with similar preferred orientations (i.e., orientation domains). We also examined direction maps in layers 2 and 3. Nearby direction-selective neurons often shared their preferred directions, although only a few neurons were direction selective in these layers.

\section{Materials and Methods}

All experimental procedures were approved by the animal experiment committee of Osaka University and conformed to the guideline of the National Institutes of Health (1996).

Animal preparation. The subjects were four macaque monkeys (Macaca fascicularis; body weight: $1.8-3.0 \mathrm{~kg}$, two males and two females). The monkeys were prepared for repeated recordings through an initial aseptic surgery (Tamura et al., 2004; Okamoto et al., 2011). A small craniotomy and durotomy (1-1.5 $\mathrm{mm}$ in diameter) was performed over the region to image in V1 under anesthesia with isoflurane (1-3\%), in a mixture of nitric oxide and oxygen (7:3). The exposed portion of the dura and cortex was covered with agar and a cover glass (Fig. $1 A, B$ ). During imaging, we stopped isoflurane administration and instead administered fentanyl citrate (Fentanyl; Daiichi-Sankyo; $35 \mu \mathrm{g} / \mathrm{kg} / \mathrm{h}$, i.v.) for analgesia (Popilskis and Kohn, 1997). The monkeys were then immobilized with pancuronium bromide (Mioblock; Organon, $0.04 \mathrm{mg} / \mathrm{kg} / \mathrm{h}$, i.v.) to prevent eye movements, and artificially ventilated with a mixture of nitric oxide and oxygen. Body temperature was maintained at $37-38^{\circ} \mathrm{C}$, and end-tidal $\mathrm{CO}_{2}$ was kept at $4.0-5.5 \%$. Electrocardiogram, blood pressure, and arterial oxygen saturation levels were continuously monitored, and maintained within an appropriate physiological range. We administered phenylephrine hydrochloride and tropicamide to the eyes to relax accommodation and dilate the pupils. The corneas were covered with hard contact lenses with a pupil ( $3 \mathrm{~mm}$ in diameter) to prevent them from drying and to allow the images on the stimulus display to be focused on the retina. After the experiments, neostigmine methylsulfate (Vagostigmin; Shionogi, $0.1 \mathrm{mg} / \mathrm{kg}$, i.m.) was administered to aid recovery of spontaneous respiration. Ketoprofen (Megeide; Nissin Pharmaceutical, $0.8 \mathrm{mg} / \mathrm{kg}$, i.m.) was administered for postsurgery analgesia. The monkeys were then returned to their home cage. For several days, they were given antibiotics (piperacillin sodium; Toyama Chemical) and ketoprofen.

In vivo calcium imaging. We performed in vivo calcium imaging from a population of $\mathrm{V} 1$ cells down reaching a depth of $310 \mu \mathrm{m}$ below the cortical surface. A border between layer 2 and layer 3 was located $270 \mu \mathrm{m}$ below the cortical surface in a histological reconstruction from one monkey, suggesting that the recording regions were mostly in layer 2 or the uppermost tier of layer 3. We injected a membrane-permeable calcium-sensitive dye (Oregon Green 488 BAPTA-1 AM or OGB-1; Invitrogen) at a depth of 250-300 $\mu \mathrm{m}$ from the cortical surface through a micropipette by air pressure using Picospritzer III (General Valve; Fig. 1B). OGB-1 (0.3 mM) was dissolved in a solution containing $0.2 \%$ Pluronic F-127 (Invitrogen), 2.5\% dimethyl sulfoxide (Sigma-Aldrich), 10 mм HEPES, $2.5 \mathrm{~mm} \mathrm{KCl}$, $150 \mathrm{~mm} \mathrm{NaCl}, 2.0 \mathrm{~mm} \mathrm{CaCl}_{2}$, and $1.0 \mathrm{~mm} \mathrm{MgCl}_{2}, \mathrm{pH}$ 7.4. In some experiments, sulforhodamine 101 (SR101; $100 \mu \mathrm{M}$, Invitrogen) was injected together with OGB-1 for identification of astrocytes (Nimmerjahn et al., 2004; Fig. 1C). In the other experiments, when we performed cellattached recording of single neuron responses, we did not use SR101 to better visualize glass pipettes loaded with a dye having an overlapping range of fluorescence wavelength (Alexa Fluor 594, see below for details). Fluorescence was imaged with a two-photon microscope customized for use in monkeys (Prairie Technologies) equipped with a mode-locked Ti:sapphire laser (MaiTai; Spectra-Physics) and a waterimmersion objective lens (LUMPlanFL40xW/IR, NA 0.8; Olympus). With this configuration of our system, blur in the $z$-direction (SD of the normal distribution function fitted to the fluorescence from a $0.1 \mu \mathrm{m}$ microsphere in the $z$-direction) was $1.5 \pm 0.15 \mu \mathrm{m}(n=13$, mean $\pm \mathrm{SD})$. One pixel corresponded to $1.09 \times 1.09 \mu \mathrm{m}^{2}$ in $x, y$-coordinates. We scanned a square region of $279 \times 279 \mu \mathrm{m}^{2}(256 \times 256$ pixels $)$ at the maximum. The frame rate was within a range of 1.7-2.8 frames/s, negatively proportional to the area of the imaged region.

Cell-attached recording. For cell-attached recording from OGB-1labeled neurons, we inserted a glass micropipette (impedance: 4-8 M $\Omega$ at $1 \mathrm{kHz}$ ), filled with $5.0 \mu \mathrm{M}$ Alexa Fluor 594 hydrazide, $10 \mathrm{~mm}$ HEPES, $2.5 \mathrm{~mm} \mathrm{KCl}, 150 \mathrm{~mm} \mathrm{NaCl}, 2.0 \mathrm{~mm} \mathrm{CaCl}_{2}$, and $1.0 \mathrm{~mm} \mathrm{MgCl}_{2}, \mathrm{pH} 7.4$, 
into the imaged cortical regions (see Fig. 2A). While advancing the pipette to a labeled neuron, we continuously ejected the solution from the tip with positive air pressure. When the electrode tip was sufficiently close to a targeted cell, dye diffusion weakened (Kitamura et al., 2008). Then, we stopped ejection of the dye and applied negative air pressure to establish cell-attached recording. Voltage signals were amplified ( $\times 10,000$, band-passed: $500-2000 \mathrm{~Hz}$ ), digitized $(20 \mathrm{kHz}, 12 \mathrm{bit})$, and stored on a computer. At the end of each recording, we verified that the recorded neuron was the one we targeted by breaking the membrane of the neuron and injecting the dye.

Visual stimulation. Visual stimuli were presented to the monkey on a liquid crystal display monitor $\left(17\right.$ inch monitor: $68^{\circ} \times 54^{\circ}, 1280 \times 1024$ pixels or 3.5 inch monitor: $21^{\circ} \times 16^{\circ}, 640 \times 480$ pixels; distance between the monitor and eyes of the monkey, 28.6 and $20 \mathrm{~cm}$, respectively). The stimuli were a circular patch of black-white drifting square-wave gratings presented on a gray homogeneous background (spatial frequency: 1.0 cycles $/^{\circ}$, temporal frequency: $4 \mathrm{~Hz}$ ). The stimulus diameter was equal to the height of the monitor (i.e., $54^{\circ}$ or $16^{\circ}$ ). In some experiments, the stimuli covered the full screen. A square-wave grating has multiple spatial frequencies, increasing the likeliness of activating many neurons tuned to different spatial frequencies. The luminance of black, white, and gray were $0.1,27.8$, and $14.0 \mathrm{~cd} / \mathrm{m}^{2}$, respectively, on the 17 inch monitor, and $2.09,393$, and $213 \mathrm{~cd} / \mathrm{m}^{2}$, respectively, on the $3.5 \mathrm{inch}$ monitor. The gratings were stationary for $1 \mathrm{~s}$ after the presentation onset, and then drifted for 3-7 s. The interstimulus interval was $4-8 \mathrm{~s}$. The stimuli were drifted in 16 directions orthogonally to eight orientations at regular intervals of $22.5^{\circ}$. Each stimulus orientation condition was repeated $30-60$ times. Because the visual stimuli were achromatic, neurons that are responsive only to chromatic gratings without luminance contrast should have been excluded from our data.

Data analysis. To quantify calcium responses for single neurons, we first selected the cell body pixels manually for each imaged neuron. We then averaged the fluorescence signals across the pixels of each neuron. We did not use pixels near the rim of the cell, because they were usually dark and possibly susceptive to contamination of fluorescence from outside the cell body. The number of pixels used for individual cells was not correlated with the orientation tuning strength $\left(r_{s}=0.014, p=0.64\right.$, Spearman's rank correlation coefficient). The ratio $(d F / F)$ of the stimulusevoked change in fluorescence signal $(d F)$ to the basal level of the fluorescence signal $(F)$ during the prestimulus period (1.6-3.5 s) was measured as calcium responses to visual stimuli. When we analyzed the orientation tuning of neurons, we pooled the responses to the two gratings with the same orientation drifting in the opposite directions. Neurons that showed signal changes to at least one orientation $(p<0.05$, signed rank test with Bonferroni correction) were classified as visually responsive. Neurons that varied in the magnitude of their calcium responses according to the grating orientation ( $p<0.05$, Kruskal-Wallis test) were defined as orientation selective. For orientation-selective cells, we determined the preferred orientation by fitting responses with a circular normal distribution function (von Mises distribution function; Batschelet, 1981; Swindale, 1998). Only cells with a good fit (goodness-of-fit, $R^{2} \geq 0.7$ ) were assigned a preferred orientation. A stricter criterion for orientation selectivity ( $p<0.01$, Kruskal-Wallis test) did not affect the overall conclusion reported in this paper.

The strength of orientation tuning was measured with a circular variance $(\mathrm{CV})$ of the responses. The $\mathrm{CV}$ was calculated as follows:

$$
\text { Circular variance }(C V)=1-\left|\frac{\sum_{k} r_{k} \exp \left(i 2 \theta_{k}\right)}{\sum_{k} r_{k}}\right| \text {, }
$$

where $k$ is a stimulus number and $r_{k}$ is the magnitude of the responses $(d F / F)$ to orientation, $\theta_{k}$, and $i$ is an imaginary unit. Because the denominator is the sum of the responses to all orientations, negative responses to particular orientations (i.e., darker fluorescence during stimulus presentations) could make the denominator zero, resulting in an incorrect evaluation of orientation selectivity. We avoided this by adding a constant to all response values so that the minimum response became zero. The rationale for this manipulation is as follows. Fluorescence in a cell would take the minimum, when the cell fires no action potentials. Cortical neurons usually exhibit spontaneous (ongoing) firing at some rates, and their fluorescence is above the minimum. Negative responses in fluorescence thus reflect firing at a rate lower than the spontaneous firing rate. We assume a linear relationship between the degree of darkening in fluorescence and a decrease in the firing rate below spontaneous level. This assumption is based on the observation that darkening in some neurons was graded and stronger for null orientation. However, how well the negative responses linearly reflect decreases in the firing rate is unknown, and it probably differs from neuron to neuron. We therefore performed another analysis by defining the magnitude of any negative (i.e., darkening) responses as zero. This manipulation was based on an assumption that if the fluorescence is reduced below a baseline cells with a low firing rate are being completely suppressed. The new procedure did not alter our conclusions regarding the relationships between orientation selectivity and local map structure. The distributions of the CVs obtained from the two calculation were not different from each other $(p=0.15$, two-sample Kolmogorov-Smirnov test). This is probably because the absolute magnitude of fluorescence at the bottom of tuning curves was only $14 \%$ (median) of the fluorescence at the peak.

We quantified the degree of diversity of the orientation preferences of neurons around a given pixel by calculating a local heterogeneity index as follows:

$$
\begin{array}{r}
\text { Local heterogeneity index }=1-\left|\frac{\sum_{n} D_{n} \exp \left(i 2 \theta_{n}\right)}{\sum_{n} D_{n}}\right|, \\
D_{n}=\exp \left(-\frac{d_{n}^{2}}{2 \sigma^{2}}\right),
\end{array}
$$

where $n$ is a neuron identity number, $d_{n}$ is the distance between a given pixel and the neuron $n, \theta_{n}$ is the preferred orientation of the neuron $n$, and $\sigma$ equals to $50 \mu \mathrm{m}$. The value of $50 \mu \mathrm{m}$ for $\sigma$ was chosen so that $D_{n}$ approximates the connection probability as a function of distance between two cortical neurons (Holmgren et al., 2003; Ashby and Isaac, 2011). Use of $\sigma$ equal to $75 \mu \mathrm{m}$ did not alter our conclusions regarding the relationships between orientation selectivity and local map structure. For calculation of the local heterogeneity index we used only orientationselective neurons with a good fit of orientation tuning $\left(R^{2} \geq 0.7\right)$. The median CV was 0.69.

We examined the relationship between the orientation tuning strength of individual cells and the variations in orientation preferences in a local region by calculating correlations between a neuron's $\mathrm{CV}$ and local heterogeneity index at the neuron's location. A technical concern in this analysis is that fitting the data with a tuning function can potentially affect the correlation. If preferred orientations are estimated less accurately for neurons with weaker orientation tuning (high CV) than for neurons with stronger orientation tuning (low CV), preferred orientations may be estimated to be more heterogeneous in a region with weaker tuning than in a region with stronger tuning. This potentially leads to a spurious correlation between orientation tuning strength and heterogeneity of preferred orientations in a local region. We therefore evaluated the confidence intervals of correlation between the $\mathrm{CV}$ and local heterogeneity index by performing a permutation test. We calculated the fitting residuals by subtracting the fitted function from the original data, shuffled the residuals, added the shuffled residuals to the fitted function, and then re-estimated preferred orientations of the same neuron. Then, we calculated local heterogeneity index, re-estimated the correlation between the index and CV of neurons, and repeated this process 1000 times to obtain the confidence interval (2.5-97.5\%) of the correlation.

For neurons assigned preferred orientation, we further analyzed direction selectivity by comparing the responses to the two opposite directions orthogonal to the preferred orientation. Direction selectivity was calculated as follows:

$$
\text { Direction selectivity }=\frac{r_{\text {prefdir }}-r_{\text {oppdir }}}{r_{\text {prefdir }}+r_{\text {oppdir }}},
$$

where $r_{\text {prefdir }}$ and $r_{\text {oppdir }}$ are the response magnitudes for the preferred and null directions, respectively. The response magnitudes were estimated from a tuning curve fitted with circular normal distribution functions, not from the raw data. 
A
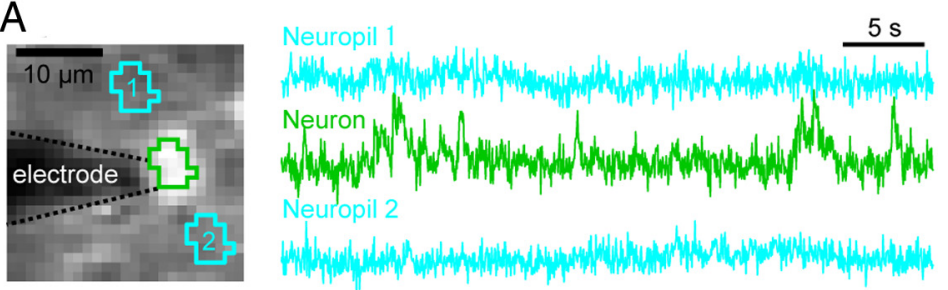

B
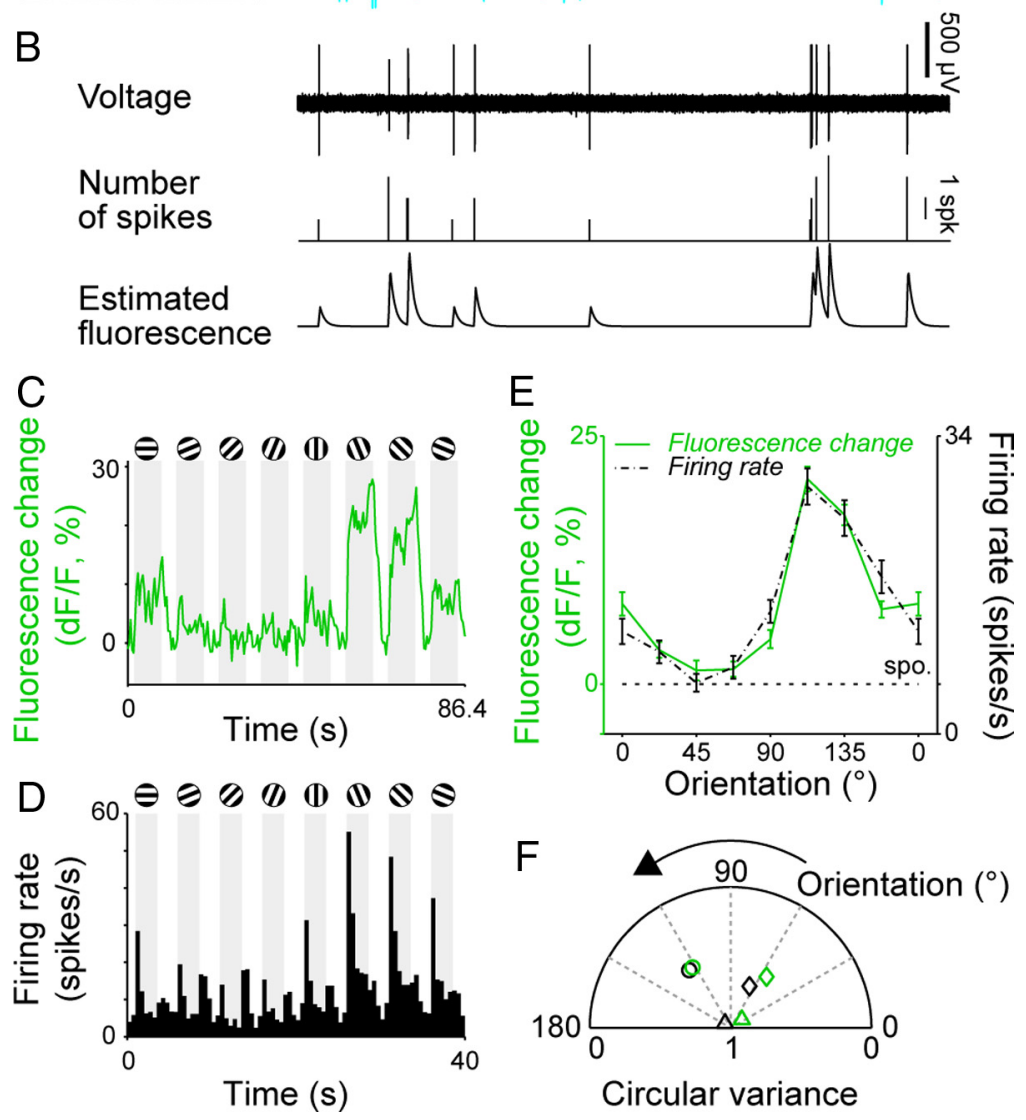

Figure 2. Relationship between fluorescence signals and spiking activities of neurons. $A$, Fluorescence signals from a V1 neuron and two nearby regions. Left, A structural image during recording. The brighter portion in the center is the recorded neuron. The darker portion to the left of the neuron is the recording electrode. Right, Fluorescence signals over a period of $35 \mathrm{~s}$ with no visual stimulation. Fluorescence signal of a neuron (second row) was extracted from the region enclosed by green lines seen in the left part. Blue traces (first and third rows) were fluorescence signals from nearby neuropils enclosed by blue lines seen in the left part. $\boldsymbol{B}$, Upper, Voltage signal obtained from the neuron simultaneously recorded with fluorescence signal. Middle, A histogram showing the number of spikes in one time bin (time bin width $=38 \mathrm{~ms}$ ). Bottom, Estimated fluorescence from spiking activities. $\boldsymbol{C}, \boldsymbol{D}$, Fluorescence change $(d F / F)$ and firing rate (poststimulus time histogram) of another $V 1$ neuron in response to gratings of various orientations. Responses were averaged across 60 stimulus presentations for the fluorescence change and 30 stimulus presentations for the firing rate. Shading indicates periods of stimulus presentation. $\boldsymbol{E}$, Orientation tuning curves based on the fluorescence signal amplitude $(\boldsymbol{C})$ and the spike firing rate $(\boldsymbol{D})$. Error bars indicate SEM. $\boldsymbol{F}$, Preferred orientation and tuning strength of three neurons (denoted by 3 different symbols) determined from fluorescence signal (green) and spike firing rate (black). CV and preferred orientation were shown as radius and azimuth in a circular plot, respectively.

Database. We performed imaging in 10 planes in five hemispheres of four animals. Four planes had the same tangential position with different cortical depths (170, 197, 240, and $265 \mu \mathrm{m}$ below the pia mater; see Fig. $6 A-D)$. Two planes overlapped in $40 \%$ of their imaged plane in the tangential dimension, but had different cortical depths (175 and 192 $\mu \mathrm{m})$. In the 10 planes, 1333 cells were loaded with OGB-1. In 5 of the 10 planes, we used SR101 for labeling astrocytes. Cells (610) were loaded only with OGB-1, 73 cells were loaded only with SR101, and 38 cells were double-labeled with OGB-1 and SR101. We classified the 610 cells loaded with OGB-1, but not SR101, as neurons and included them in our analysis. The 38 double-labeled cells were classified as astrocytes (Nimmerjahn et al., 2004) and excluded from the analysis described (see Results). Twenty-nine of these cells showed increased fluorescence signals in re- sponse to visual stimuli, and eight of them were orientation selective. The other 685 cells in the five planes without SR101 administration included both neurons and astrocytes. Based on SR101 staining in the other planes, we estimated the contamination rate of astrocytes to neurons as $\sim 6 \%$. Of the $1295(610+685)$ neurons we recorded, 1106 (85\%) showed responses to at least one orientation. Of these 1295 neurons, 869 neurons (67\%) showed orientation selectivity. Preferred orientations were determined for 548 of the 869 neurons $(63 \%)$ among orientation-selective neurons.

\section{Results}

Fluorescence signal and spiking activity of single neurons

We first addressed how faithfully the preferred orientation and strength of orientation tuning determined by calcium imaging reflected those represented by action potentials. Because the spatial resolution of two-photon imaging is relatively low in the $z$-direction, fluorescence signals from neuropils above and below an examined cell body may possibly contaminate fluorescence signals recorded from the cell body (Sato et al., 2007). If this happens, the contamination leads to an inaccurate estimation of orientation tuning curves. Fluorescence signals also saturate when the firing rate is high, which potentially leads to an estimation of broader orientation tuning (Nauhaus et al., 2012a).

We examined how well calcium signals of cell bodies reflected firing of action potentials (spikes) by recording from a single neuron in an imaged region using cellattached recording techniques (Fig. 2A). Fluorescence was imaged at a sampling rate of $25 \mathrm{~Hz}$ in this experiment. Every spike was followed by a calcium transient (Fig. 2, compare green trace in $A$ with upper trace in $B$ ). When spikes occurred in bursts (Fig. 2B, middle trace), calcium transients became larger and lasted longer. We estimated the fluorescence signal of the neuron by convolving a spike train with a function of the calcium signal changes that we fit to the spike-triggered average of the fluorescence changes. The estimated fluorescence signal (Fig. $2 B$, bottom) was similar to the recorded calcium signal (Fig. $2 A$, middle; $r=0.54$, $\left.p<10^{-5}\right)$. The results indicate that fluorescence signals in a cell body are a good indicator of spike occurrences at least for neurons with a low firing rate.

Transient increases in fluorescence with a rapid rise and a slow decay were observed only in the recording from the cell body (Fig. $2 A$, green traces), but not from surrounding regions, presumably neuropils (Fig. $2 A$, blue traces), indicating that fluorescence of cell bodies is unlikely recorded from surrounding regions or vice versa.

We compared orientation tuning curves obtained from spikes with those obtained by fluorescence signals sampled at $1.7 \mathrm{~Hz}$. 
Figure $2 C$ shows fluorescence change in a neuron in response to eight different orientations, and Figure $2 D$ shows a concatenated peristimulus time histogram of the spike responses of the same neuron. The two orientation tuning curves obtained from these data were similar in shape (Fig. $2 E)$. The preferred orientation based on fluorescence signals matched to that based on spikes $\left(126^{\circ}\right.$ for calcium signal and $122^{\circ}$ for spikes). The $\mathrm{CV}$ derived from the spike response (0.50) closely matched to the one calculated from fluorescence signals $(0.55)$. In a population of three neurons, the orientation tuning calculated from fluorescence signals matched to the one calculated from spike responses for both preferred the orientation and tuning strength (Fig. $2 F$ ), suggesting that the preferred orientations and the orientation tuning strength obtained from fluorescence signals can be used to estimate those in spikes. Because we did not optimize stimulus parameters such as size, spatial frequency, and temporal frequency for individual cells, our cells may not have been activated strongly, maintaining the linearity between the firing and fluorescence signals.

\section{Orientation preference map}

Using in vivo two-photon imaging techniques, we measured calcium-signal responses to oriented gratings simultaneously from a large number of neurons in a local region of V1. In an imaged region of $279 \times$ $279 \mu \mathrm{m}$ (Fig. 3), we analyzed responses from a total of 227 neurons. As expected from the well established orientation column structure in V1, nearby neurons exhibited a strong tendency to prefer similar stimulus orientations. For example, neurons labeled as a-c in Figure $3 A$ were located within 75 $\mu \mathrm{m}$ of each other, and all responded the most to near horizontal orientations (Fig. $3 B, C$ ). Optimal orientations determined from fitted tuning curves were $177^{\circ}$ for neuron $a, 160^{\circ}$ for neuron $b$, and $173^{\circ}$ for neuron c. For the entire population of 227 cells, cumulative plots of differences in preferred orientations between two cells shifted to the right with physical distance between them (Fig. 3D), indicating that differences in preferred orientation increased with physical separation. The distance between two cells across the cortex and the difference in their preferred orientations was positively correlated $\left(r_{s}=0.41, p<10^{-5}\right.$, Spearman's rank correlation coefficient). This was the case for all of the 10 imaged sites $(p<0.05$, Spearman's rank correlation coefficient). The mean of the correlation coefficients for the 10 imaged regions was $0.30 \pm 0.14$ (mean \pm $\mathrm{SD})$.

Based on the orientation tuning of individual cells, we derived an orientation preference map at the level of single cells. Figure $3 C$ shows the spatial arrangement of preferred orientations determined for individual neurons in the imaged region. Most neurons in the upper imaging field preferred orientation gratings of $\sim 135^{\circ}$ (leftward tilted orientation), those in the middle third of the field preferred $0^{\circ}\left(180^{\circ}\right)$, and those in the lower field preferred orientation
B

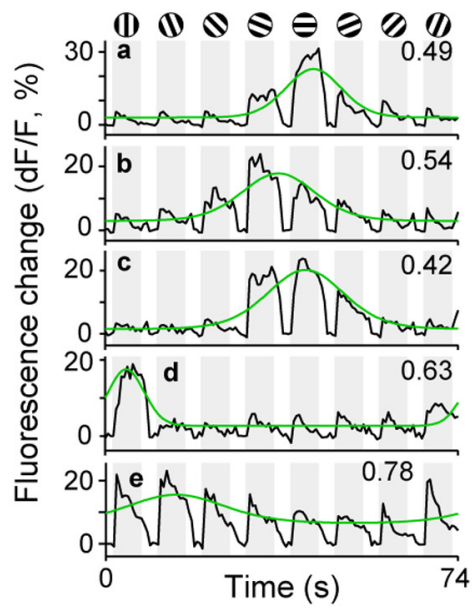

$50 \mu \mathrm{m}$

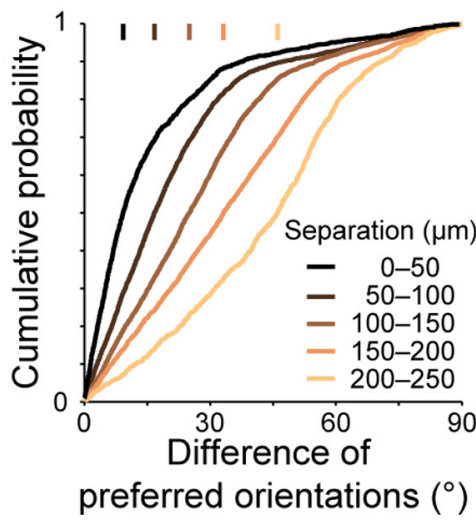

Figure 3. Orientation preference map obtained by two-photon calcium imaging. $A$, A recording region loaded with $0 G B-1$. The image was taken in layer 2 in a tangential plane $145 \mu \mathrm{m}$ below the cortical surface. $\boldsymbol{B}$, Time courses of fluorescence changes ( $d F / F)$ tuning curves $\left(R^{2}<0.7\right)$. Open circles: visually responsive, orientation nonselective cells. $\boldsymbol{D}$, Relationship between the difference in the preferred orientations of two neurons and the physical distance between them in the cortex. Vertical bars: the median values of differences of preferred orientation for five groups of neuron pairs with different physical separations.

gratings of $30^{\circ}$ (Fig. 3C). The preferred orientation of orientationselective domains gradually shifted counterclockwise over the imaged region. It should be noted that there were clusters of neurons with no or poor orientation selectivity among these orientationselective neurons (denoted by circles or dots).

Detailed inspection, however, revealed that some areas in the imaged regions contained neurons with preferred orientations markedly different from those of their neighbors. For example, neurons labeled as $\mathrm{d}$ and $\mathrm{e}$ in Figure $3, A$ and $B$, were tuned to vertical orientations $\left(82^{\circ}\right.$ for neuron $d, 107^{\circ}$ for neuron e), whereas most of the surrounding neurons were tuned to horizontal orientations. There was a slow saturation in the upper right part of the cumulative plot for the case of $0-50 \mu \mathrm{m}$ separations, indicating that nearby neurons occasionally preferred markedly different orientations (difference of orientations $>45^{\circ}$; Fig. $3 D$, black curve). The degree of homogeneity of orientation preference in a local region thus varied across the cortical surface.

The preferred orientations of neurons were heterogeneous around the center of the imaged region shown in Figure $4 A$, whereas most of the cells located in the upper right and lower left portions shared similar preferred orientation. Preferred orientations between the upper right and lower left section were approximately orthogonal to each other. In Figure $4 B$, we show the distribution of the 
A

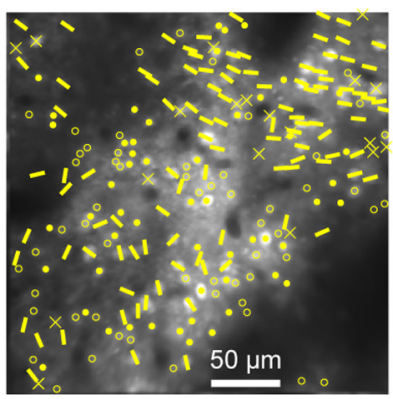

C

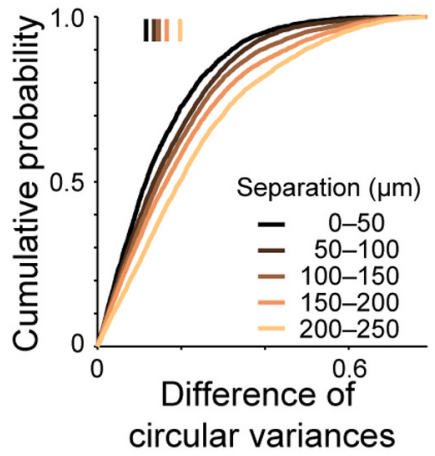

B

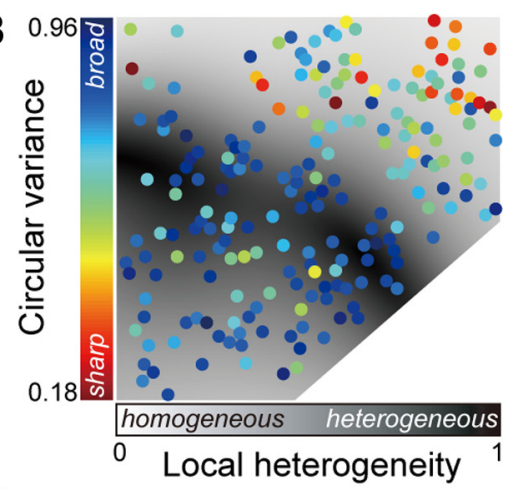

D

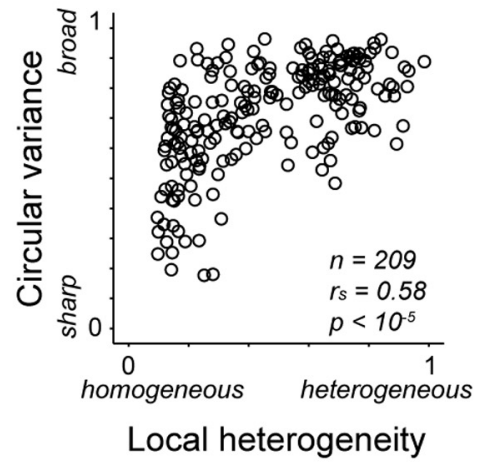

E
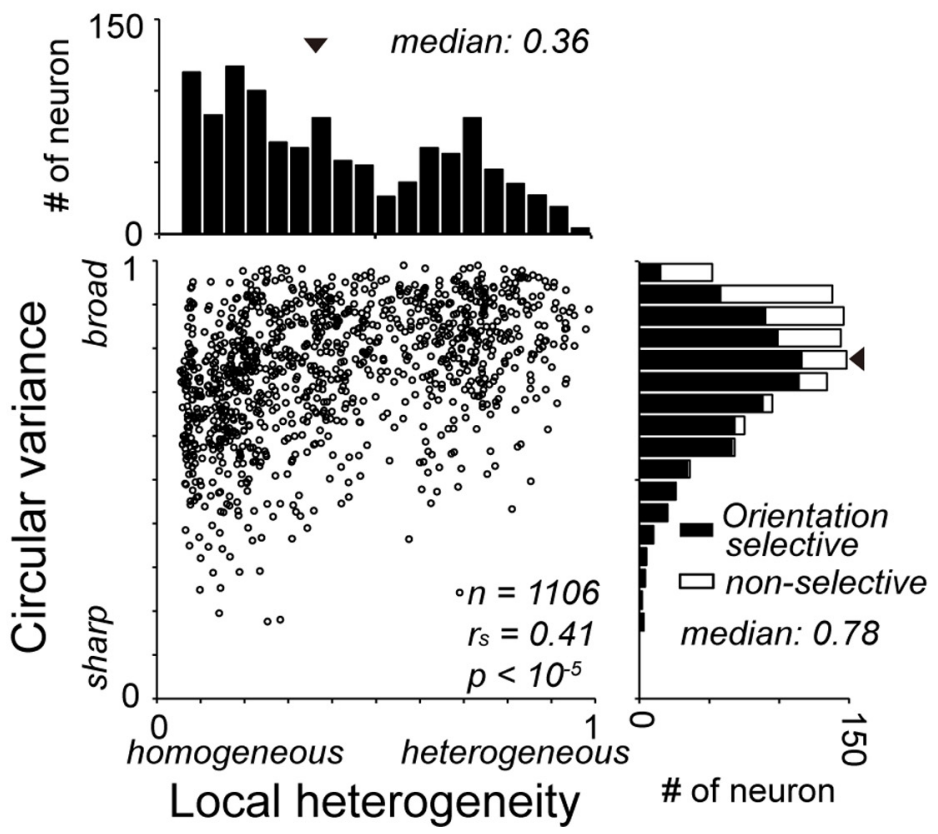

Figure 4. Arrangement of neurons according to their orientation tuning strength. $\boldsymbol{A}$, Orientation preference map in layer 2 superimposed on a structural image. Crosses denote visually nonresponsive cells. Other conventions are the same as those in Figure 3C. $\boldsymbol{B}, A$ map of the orientation tuning strength (CV) and the local heterogeneity of preferred orientations (local heterogeneity index). The colors of dots indicate the (V of neurons at the dot positions. The gray level of background pixels indicates the local heterogeneity index. C, Cumulative plots of the difference in CV between two neurons by physical separation between the neurons. Vertical bars: median values of the differences for five groups of distances between the neurons. $\boldsymbol{D}$, Plots of CVs against local heterogeneity indices for the recording region shown in $\boldsymbol{B}$. $\boldsymbol{E}$, Population data of CVs and local heterogeneity indices for 1106 neurons in 10 imaged regions.

heterogeneity of preferred orientations by mapping the local heterogeneity index in gray scale (see Materials and Methods). For calculation of the local heterogeneity index, we used only neurons with orientation tunings that had a good fit for the circular normal distribution function (goodness-of-fit, $R^{2} \geq 0.7$ ). The frequency distri- bution of the local heterogeneity index in all imaged regions was bimodal (Fig. $4 E ; p=$ 0.0002 , Hartigan's dip test), indicating that $\mathrm{V} 1$ is composed of homogeneous regions and heterogeneous regions in respect to preferred orientations. This is consistent with single-unit recording and intrinsic signal optical imaging studies showing that V1 contains iso-orientation domains and orientation fracture or pinwheel-like structure. The dark band in the center of Figure $4 B$ indicates that there was a marked variety of preferred orientations along this line. The center portion of the imaged region appears to correspond to an orientation fracture reported in studies using intrinsic signal optical imaging (Blasdel and Salama, 1986).

\section{Orientation tuning strength map}

By calculating $C V$ values for visually responsive neurons, we generated a map of orientation tuning strength (Fig. $4 B$ ). In the region shown in Figure $4 B$, neurons with sharp orientation tuning (indicated by orange and red dots) were clustered in the upper right field, whereas neurons in the center and lower left fields showed broad orientation tunings (indicated by light and dark blue dots). A cumulative plot of difference in tuning strength between two neurons gradually shifts with physical separation between them, indicating that closer neurons have similar orientation tuning strengths than neurons spaced further apart (Fig. 4C). The physical separation between two cells across the cortex was positively correlated with the difference in their CVs $\left(r_{s}=0.19, p<10^{-5}\right.$, Spearman's rank correlation coefficient). This was true for 8 of the 10 imaged regions $(p<0.05$, Spearman's rank correlation coefficient). The mean of the correlation coefficients for the 10 imaged regions was $0.20 \pm 0.17$ (mean $\pm \mathrm{SD}$ ). Orientation tuning strength $(\mathrm{CV})$ of visually responsive cells $(n=1106)$ was not correlated with signal-to-noise ratio, a ratio of the magnitude of the largest fluorescence signal to the variance of the fluorescence signal in individual cells during the imaging period $\left(r_{s}=0.016, p=0.59\right.$; Spearman's rank correlation coefficient). These results indicate that clustering of the neurons with similar tuning strength unlikely arise from difference of quality of imaging across the cortex.

We next examined the relationship between the heterogeneity of the orientation preferences of cells in a local region and the strength of orientation selectivity of individual cells. This relationship has been examined at a larger scale using intrinsic optical imaging (Bar- 


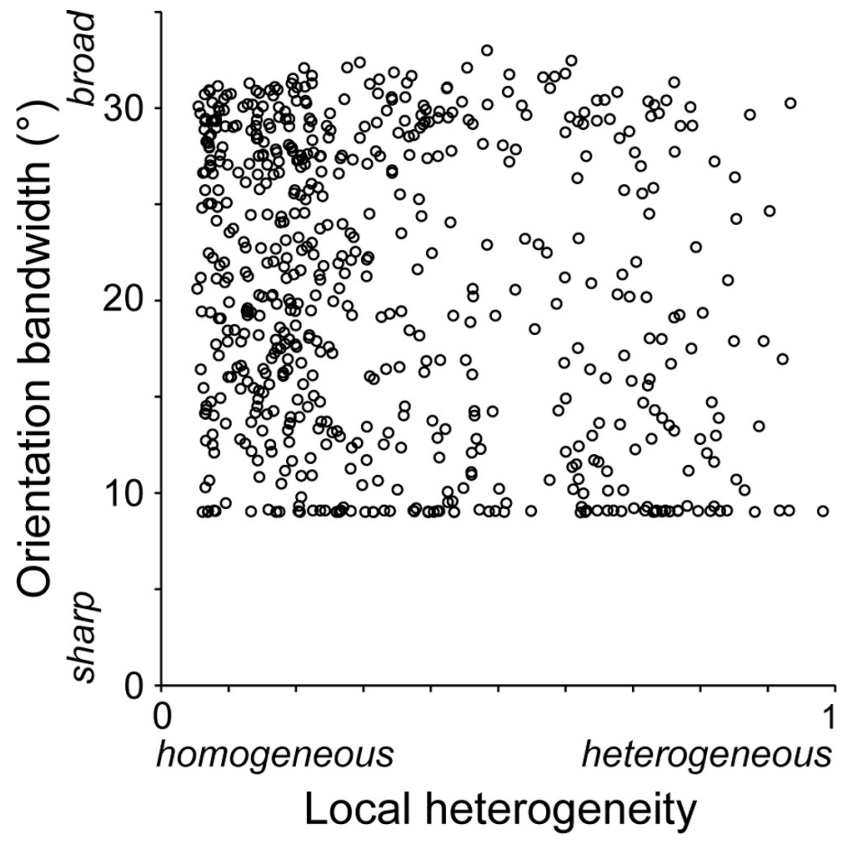

Figure 5. Relationship between orientation bandwidth of neurons and local heterogeneity index. The plots are based on 614 neurons with well fitted orientation tuning curves $\left(R^{2} \geq 0.7\right)$.

tfeld and Grinvald, 1992; Maldonado et al., 1997; Schummers et al., 2002; Mariño et al., 2005; Nauhaus et al., 2008). We found that neurons in a region of heterogeneous orientation preferences showed weak orientation selectivity (high CV), whereas the orientation selectivity of neurons in a region of homogeneous preferred orientations varied from strong to weak. In the imaged region shown in Figure $4 B$, neurons in highly heterogeneous regions around the center of the imaged plane (dark gray areas) showed broad orientation tuning (bluish dots). Neurons located in relatively homogeneous regions (light gray areas in the upper right and lower left portions) were either sharply or broadly tuned to orientation (bluish and reddish dots intermingled). For the 209 neurons shown in the imaged plane of Figure $4 A$, the CVs of these neurons were positively correlated with the heterogeneity of the orientation preferences of the surrounding neurons (Fig. $4 D ; r_{s}=0.58, p<10^{-5}$; Spearman's rank correlation coefficient). The $95 \%$ confidence interval was 0.57-0.59 (see Materials and Methods). In 7 of 10 imaged planes, CV values were positively correlated with local heterogeneity index values $\left(r_{s}=0.25-0.80, p<0.05\right)$, and the confidence interval of the correlation values did not contain 0 . Pooled data from 10 imaged planes also showed a similar correlation $\left(r_{s}=0.41, p<10^{-5}\right.$; Fig. $\left.4 E\right)$. Orientation tuning of a majority of neurons were weak with $\mathrm{CV}$ values larger than 0.5 (Fig. $4 E$, right histogram). This is probably because most of the recorded neurons were in layer 2 (Gur and Snodderly, 2008).

We also measured bandwidth of the orientation tuning curve. For each neuron, we fitted the data with a circular normal distribution function. We then measured half-width at $60.7 \%$ of peak height from baseline responses (corresponding to $\sigma$ in the normal distribution function) for 614 neurons with well fitted orientation tuning curves $\left(R^{2} \geq 0.7\right)$. We excluded 492 poorly fitted neurons $\left(R^{2}<0.7\right)$ to avoid an incorrect estimation of tuning curves. In both regions of homogeneous preferred orientations and regions of heterogeneous preferred orientations, bandwidths varied from sharp to broad (Fig. 5). We observed many neurons in heterogeneous regions had small bandwidth values. These neurons had a sharp response peak with baseline responses out- side the peak. It should also be noted that many neurons were distributed along a lower bound $\left(9^{\circ}\right)$ in a scatterplot of Figure 5. This is because the sampling interval was $22.5^{\circ}$ in our orientation tuning test, and bandwidths of sharply tuned neurons were not properly evaluated. After excluding 77 sharply tuned neurons with a bandwidth $<10^{\circ}$, no correlation between the bandwidth and the local heterogeneity index was observed $\left(r_{s}=-0.07, p=\right.$ $0.11, n=537$; Spearman's rank correlation coefficient).

The local heterogeneity index can be calculated for neurons without nearby surrounding neurons, because the weighting Gaussian function has a long tail and covers the entire imaged regions. Therefore, the index assigned to a given neuron may reflect the heterogeneity of preferred orientations of distant neurons. To better reflect the heterogeneity in orientation preferences around neurons in a local region, we modified the local heterogeneity index by changing the weighting function from a Gaussian function to a step function, $D_{n}=\left\{\begin{array}{ll}1 & \left(d_{n} \leq 50\right) \\ 0 & \text { otherwise }\end{array}\right.$. This modified heterogeneity index was correlated with orientation tuning strength $(\mathrm{CV})$ across the recorded neurons in 8 of 10 planes $\left(r_{s}=0.30-0.96, p<0.01\right)$. The results were consistent with the results derived using the original, local heterogeneity index.

\section{Clustering of neurons sharing their tuning strength across cortical depth}

We so far described the arrangement of neurons in a plane parallel to the cortical surface according to their orientation tuning strength. Here, we examined whether the clustering of neurons according to their tuning strength was preserved across the entire depth of layer 2. We obtained maps for orientation tuning strength $(\mathrm{CV})$ of four different sets of neurons at different depths $(170,197,240$, and $265 \mu \mathrm{m})$ at the same tangential position of the cortex (Fig. $6 A-D$ ). The border between layers 2 and 3 was 270 $\mu \mathrm{m}$ below the cortical surface as determined in histological reconstruction, indicating that the four planes covered the entire thickness of layer 2. In each imaged plane, neurons in an upper field had sharper orientation tuning than those in a lower field. Overlaying these maps showed that neurons at a similar tangential position shared a similar tuning strength (Fig. 6E). These results indicate that neurons with similar orientation tuning strengths are arranged vertically in layer 2 .

\section{Direction selectivity}

We found only a few neurons with a high direction selectivity index $(>0.33$; i.e., the response magnitude to the preferred direction is more than twice that of the null direction; $5.5 \pm 4.6$ cells for an imaged region, mean $\pm \mathrm{SD}$; Fig. $7 A-\mathrm{C}$ ), as has been reported by single-unit recording studies (Dow, 1974; Livingstone and Hubel, 1984; Leventhal et al., 1995; Gur and Snodderly, 2008, but see Sato et al., 1995). Figure $7 A$ shows calcium responses of a rare direction-selective cell ( $\mathrm{a}$; direction selectivity index $=0.47$ ) and a nondirection selective cell (b; direction selectivity index $=$ 0.01 ). Three imaged regions contained $>7$ neurons with a direction selectivity index of $>0.33$. In two of three regions, the difference in the preferred directions between two neurons was correlated with the physical distance between them $\left(r_{s}=0.22\right.$ and $0.49 ; p<0.05$; Fig. $7 C, D)$. In the imaged plane shown in Figure $7 C, 14$ of 16 neurons preferred the left-to-right direction.

\section{Discussion}

Using in vivo two-photon calcium imaging techniques, we examined the spatial arrangement of neurons in layer 2 and the upper- 

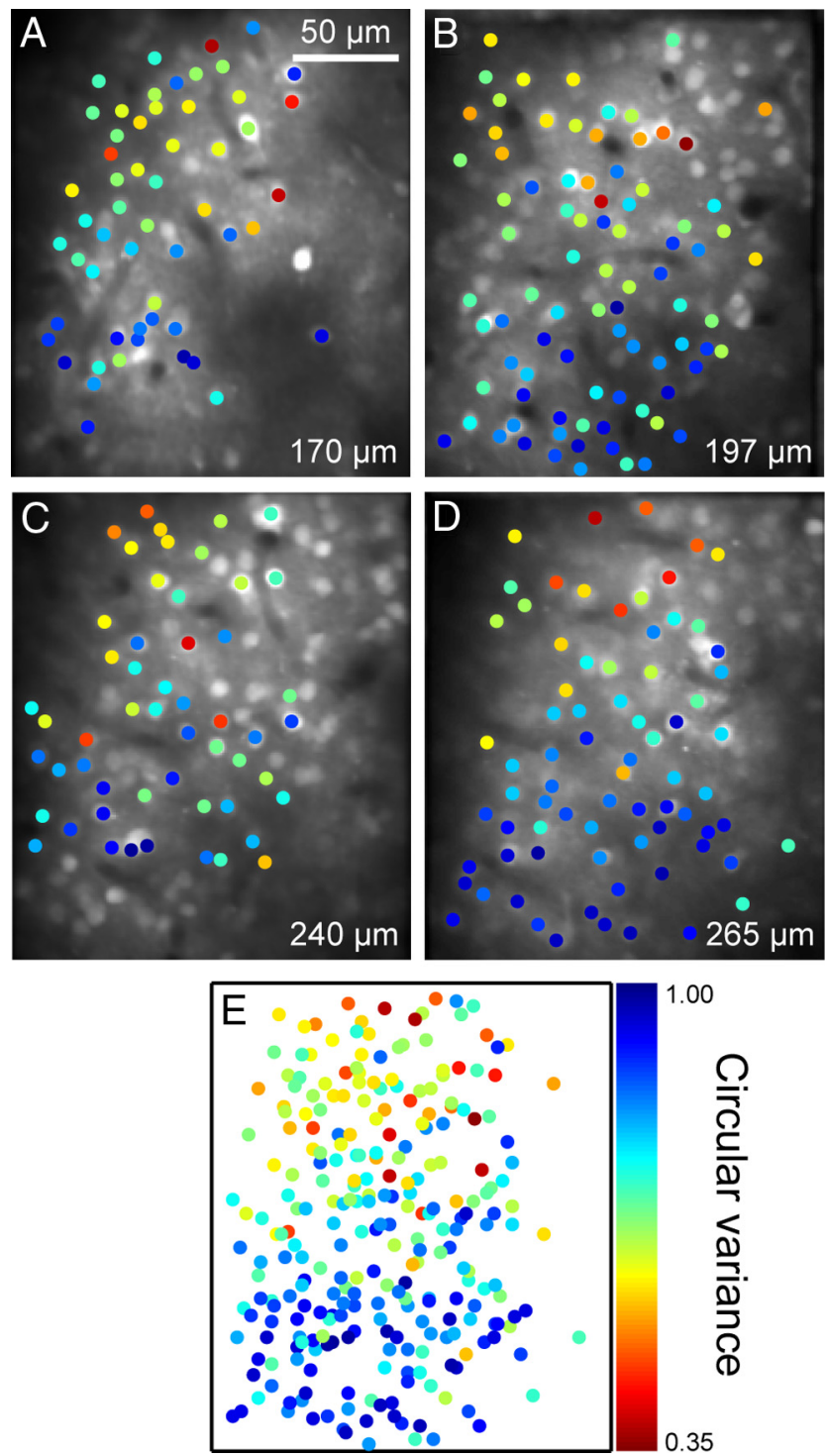

Figure 6. Consistency of orientation tuning strength maps across cortical depth. $\boldsymbol{A}-\boldsymbol{D}$, Four maps for orientation tuning strength from neurons at the same tangential positions and different cortical depths. Dot colors indicate the CVs of the neurons at that dot position. $\boldsymbol{E}$, An overlay of the four maps.

most tier of layer 3 of monkey V1 according to orientation tuning strength. Neurons with similar tuning strengths were clustered over the cortex approximately at a scale of $50 \mu \mathrm{m}$ and across at a least $100 \mu \mathrm{m}$ depth. Neurons in regions of heterogeneous orientation preferences (at orientation fractures) had weak or no orientation tuning, whereas neurons surrounded by neurons with similar preferred orientations exhibited weak to strong orientation tunings. Neurons in monkey V1 are arranged according to their orientation tuning strength, and this arrangement is related to their orientation preference map.

\section{Orientation preference map at the level of single neurons}

The preferred orientations of V1 neurons typically changed smoothly across the cortex (Fig. 3), as consistent with previous single-unit recording studies (Hubel and Wiesel, 1974; Schiller et al., 1976; Livingstone and Hubel, 1984; Leventhal et al., 1995), intrinsic signal optical imaging studies (Blasdel and Salama, 1986; Blasdel, 1992), and a two-photon calcium imaging study (Nau- haus et al., 2012b). At sporadic sites, the preferred orientations of neurons changed abruptly along a line across the cortex, forming orientation fractures (Fig. 4B). Our imaging planes did not contain a typical pinwheel center, probably because the area of the imaged regions was limited. Furthermore, some exceptional neurons in iso-orientation domains preferred orientation different by $>45^{\circ}$ from their surrounding neurons (Fig. $3 \mathrm{~B}, \mathrm{C}$ ). This scatter of preferred orientation in a local region in monkey $\mathrm{V} 1$ appears to be different from the extremely precise arrangement of orientation preferences reported for cat area 18, where there seems to be no neurons with preferred orientations deviated $>45^{\circ}$ from the mean preferred orientation of iso-orientation domain (Ohki et al., 2006). Multiple single-unit recording studies reported similar heterogeneity of preferred orientations in cat V1 (Maldonado and Gray, 1996; Hetherington and Swindale, 1999). These authors assumed that there is a smooth orientation map and that the heterogeneity of preferred orientations arises from scattering of the physiological positions of neurons sampled by tetrodes. In cat V1, this assumption seems to be correct. However, in monkey V1, even nearby neurons can sometimes prefer substantially different orientations in iso-orientation domains.

\section{Neuronal organization of orientation tuning strength}

Orientation tuning strength was similar among nearby neurons, and became increasingly different with separation between neurons, suggesting that there is an organization across V1 cortex of orientation tuning strength (Fig. 4C). Orientation tuning strength was positively correlated with the heterogeneity of preferred orientations in a local region (Fig. $4 D, E$ ). Because local heterogeneity of preferred orientation is high in orientation fractures and pinwheel centers, this result supports the previous findings that orientation tuning strength is weaker in these structures than in iso-orientation domains (Nauhaus et al., 2008; Ohki et al., 2006; but see Bartfeld and Grinvald, 1992; Maldonado et al., 1997). Nauhaus et al. (2008) found a correlation between the local variety of orientation preferences and the orientation bandwidth; neurons in iso-orientation domains show narrow bandwidths, and those in heterogeneous regions show broad bandwidths. We found a wide variability in CVs within isoorientation domains (Fig. 4E), instead of the expected finding of high CV cells only in heterogeneous regions. However, these observations may not necessarily be discrepant. CVs evaluate tuning strength over an entire range of orientations, whereas bandwidths measure tuning strength locally around a tuning peak. Neurons with sharp peaks containing baseline responses outside the peak exhibit a large CV but small bandwidth. To compare the two studies more directly, we also analyzed the bandwidth of our neurons (Fig. 5). We found no correlation between the local heterogeneity of orientation preferences and the bandwidth of individual neurons. A possible reason for this discrepancy may be that weakly tuned curves tend to be poorly fit and are not subject to measurement of bandwidths. We excluded 492 neurons (44\%) from 1106 visually responsive neurons from our bandwidth analysis. However, one problem with this is that exclusion is not random in terms of tuning strength. Most of the discarded neurons are weakly orientation selective (high in $\mathrm{CV}$; median $\mathrm{CV}=$ $0.86)$. Furthermore, we excluded another population of 77 neurons with a sharp tuning peak ( $<10^{\circ}$ width), because our sampling interval $\left(22.5^{\circ}\right)$ did not allow an estimation of bandwidth. Thus, we reached a similar conclusion to that of Nauhaus et al. (2008) that the tuning strength and local variety of orientation preferences are related, but this conclusion is based on the results of CVs, not bandwidths. 

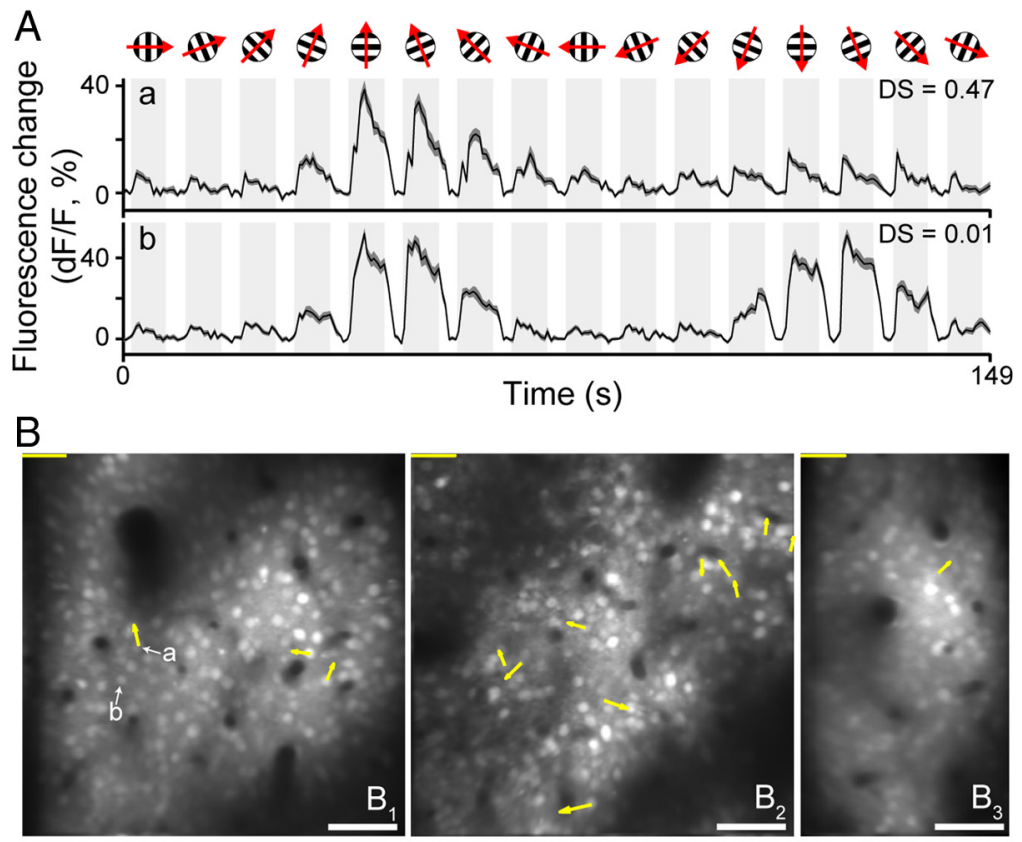

C

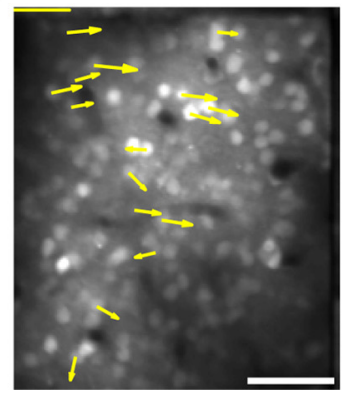

D

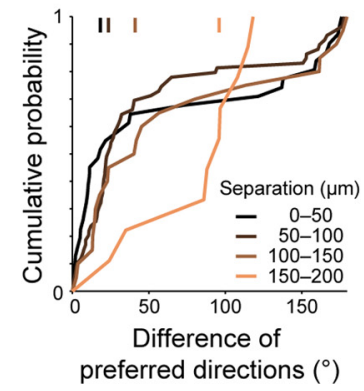

Figure 7. Direction maps in layer 2 or layer 3 obtained by two-photon calcium imaging. $\boldsymbol{A}$, Time course of fluorescence responses to drifting gratings in two representative neurons (denoted as a and $b$ in $\boldsymbol{B}$ ). Shades along the traces indicate SEM. $\boldsymbol{B}, \boldsymbol{C}$, Direction maps. Angle and length of an arrow indicate preferred direction and strength of direction tuning of a neuron with $>0.33$ of direction selectivity. Lengths of scale bars at upper left corner indicate direction selectivity 1.0. Scale bars at lower right corner: $50 \mu \mathrm{m}$. D. Relationship of difference of preferred directions of two neurons to the physical distance between them over the cortex. Data are taken from an imaged region in $\boldsymbol{C}$.

We defined a "local" region as an area of $50-75 \mu \mathrm{m}$ in radius (the $\sigma$ of the Gaussian function for weighting used in the calculation of heterogeneity). This size is similar to the sampling radius of a tetrode (Gray et al., 1995). Previous tetrode-recording studies in cat area 17 reported inconsistent results as to whether orientation tuning strength is correlated with the heterogeneity of preferred orientations. Hetherington and Swindale (1999) reported that orientation tuning width is positively correlated with the preferred orientation scatter, whereas Maldonado and Gray (1996) reported no relationship between them. The inconsistency in these tetrode studies may have arisen from a sampling bias because of the point sampling of the electrodes across the cortex. The relationship between orientation tuning strength and heterogeneity of preferred orientation in a region with $50-75 \mu \mathrm{m}$ radius suggests that orientation nonselective lateral connections between neurons at this scale in the supragranular layer weaken the orientation selectivity of neurons in heterogeneous regions, as shown in the orientation tunings in membrane potential inputs to neurons located near pinwheel centers (Schummers et al., 2002). We do not reject the possibility that there are connections between neurons that prefer similar orientations in heterogeneous regions as reported in rodent visual cortex (Ko et al., 2011; Li et al., 2012). These connections may maintain orientation selectivity in heterogeneous regions in competitive manner with orientation nonselective lateral connections. Although we assume that lateral local interaction between neurons within the supragranular layer of $\mathrm{V} 1$ underlie this relationship, it could arise from local interaction in more peripheral neural circuits, such as the granular layer of $\mathrm{V} 1$, or from local pooling of feedforward inputs from the retina or the lateral geniculate nucleus. Pooling of outputs of $\mathrm{ON}$ and $\mathrm{OFF}$ retinal ganglion cells with Gaussian-shape weighting function across the retina may underlie the orientation tuning of V1 neurons and the relationship between orientation tuning strength and local heterogeneity of preferred orientations (Ringach, 2007).

Imaging of intrinsic optical signals has shown that there are regions with low orientation selectivity in monkey $\mathrm{V} 1$; these regions include pinwheel centers and $\mathrm{CO}$ blobs (Blasdel, 1992; Lu and Roe, 2008). Because optical imaging measures spatially averaged signals from a region of the cortex of $250 \mu \mathrm{m}$ diameter (Polimeni et al., 2005), low orientation-selective regions can result from clustering of weakly orientation-tuned neurons with similar or various preferred orientations or clustering of sharply orientation-tuned neurons with various preferred orientations. We showed that orientation selectivity of a majority of neurons was weak in regions where preferred orientations were heterogeneous, suggesting that weakly orientation-selective regions in optical imaging result at least from clustering of weakly orientation-selective neurons. In CO-blobs, orientation selectivity of neurons is weaker than that of interblob neurons (Livingstone and Hubel, 1984; Economides et al., 2011). To reveal the orientation preference maps in CO-blobs, two-photon $\mathrm{Ca}^{2+}$ imaging in CO-blobs is required. Unfortunately, we failed to stain $\mathrm{CO}$-blobs of our animals by postmortem histochemistry. It remains unclear how this organization of orientation tuning strength relates to CO-blobs. Because our histochemical protocol worked for brain tissues from intact animals, the failure of staining in the experimental subjects likely resulted from long-lasting exposure to visual stimulation, which led to exhaustion of CO activity in V1.

Using two-photon calcium imaging, Ohki et al. (2006) reported that preferred orientations of neurons change precisely and systematically around pinwheel centers in area 18 of cats, and neurons were orientation selective but slightly weaker near the pinwheel centers compared with regions far from the pinwheel center. Because we did not find unequivocal pinwheel centers in our imaged regions, the orientation tuning strength and arrangement of neurons around pinwheel centers remains unclear in monkey $\mathrm{V} 1$. If the orientation tuning strength is related to heterogeneity of preferred orientations near pinwheel centers like the V1 regions in which we made recordings, most of neurons around pinwheel centers may be weakly selective or nonselective for orientation in monkey V1, unlike cat V1. 
In conclusion, V1 neurons of close proximity in layers 2 and 3 shared their orientation tuning strength and orientation preference. The functional maps for orientation tuning strength and orientation preference are related to each other. Orientation tuning is weak in a region heterogeneous for orientation preferences, whereas neurons with sharp orientation tuning are found only in iso-orientation domains consisting of neurons with shared orientation preferences. Thus, the orientation tuning strength of a neuron is partially predictable from its location in the orientation preference map at the level of single neurons.

\section{References}

Ashby MC, Isaac JT (2011) Maturation of a recurrent excitatory neocortical circuit by experience-dependent unsilencing of newly formed dendritic spines. Neuron 70:510-521. CrossRef Medline

Bartfeld E, Grinvald A (1992) Relationships between orientation-preference pinwheels, cytochrome oxidase blobs, and ocular-dominance columns in primate striate cortex. Proc Natl Acad Sci U S A 89:11905-11909. CrossRef Medline

Batschelet E (1981) Circular statistics in biology. London: Academic.

Blasdel GG (1992) Orientation selectivity, preference, and continuity in monkey striate cortex. J Neurosci 12:3139-3161. Medline

Blasdel GG, Salama G (1986) Voltage-sensitive dyes reveal a modular organization in monkey striate cortex. Nature 321:579-585. CrossRef Medline

De Valois RL, Yund EW, Hepler N (1982) The orientation and direction selectivity of cells in macaque visual cortex. Vision Res 22:531-544. CrossRef Medline

Dow BM (1974) Functional classes of cells and their laminar distribution in monkey visual cortex. J Neurophysiol 37:927-946. Medline

Economides JR, Sincich LC, Adams DL, Horton JC (2011) Orientation tuning of cytochrome oxidase patches in macaque primary visual cortex. Nat Neurosci 14:1574-1580. CrossRef Medline

Gray CM, Maldonado PE, Wilson M, McNaughton B (1995) Tetrodes markedly improve the reliability and yield of multiple single-unit isolation from multi-unit recordings in cat striate cortex. J Neurosci Methods 63:43-54. CrossRef Medline

Gur M, Snodderly DM (2008) Physiological differences between neurons in layer 2 and layer 3 of primary visual cortex (V1) of alert macaque monkeys. J Physiol 586:2293-2306. CrossRef Medline

Hetherington PA, Swindale NV (1999) Receptive field and orientation scatter studied by tetrode recordings in cat area 17 . Vis Neurosci 16:637-652. Medline

Holmgren C, Harkany T, Svennenfors B, Zilberter Y (2003) Pyramidal cell communication within local networks in layer $2 / 3$ of rat neocortex. J Physiol 551:139-153. CrossRef Medline

Hubel DH, Wiesel TN (1968) Receptive fields and functional architecture of monkey striate cortex. J Physiol 195:215-243. Medline

Hubel DH, Wiesel TN (1974) Sequence regularity and geometry of orientation columns in the monkey striate cortex. J Comp Neurol 158:267-293. CrossRef Medline

Kitamura K, Judkewitz B, Kano M, Denk W, Häusser M (2008) Targeted patch-clamp recordings and single-cell electroporation of unlabeled neurons in vivo. Nat Methods 5:61-67. Medline

Ko H, Hofer SB, Pichler B, Buchanan KA, Sjöström PJ, Mrsic-Flogel TD (2011) Functional specificity of local synaptic connections in neocortical networks. Nature 473:87-91. CrossRef Medline

Lennie P, Krauskopf J, Sclar G (1990) Chromatic mechanisms in striate cortex of macaque. J Neurosci 10:649-669. Medline

Leventhal AG, Thompson KG, Liu D, Zhou Y, Ault SJ (1995) Concomitant sensitivity to orientation, direction, and color of cells in layers 2, 3, and 4 of monkey striate cortex. J Neurosci 15:1808-1818. Medline

Li Y, Lu H, Cheng PL, Ge S, Xu H, Shi SH, Dan Y (2012) Clonally related visual cortical neurons show similar stimulus feature selectivity. Nature 486:118-121. Medline

Livingstone MS, Hubel DH (1984) Anatomy and physiology of a color system in the primate visual cortex. J Neurosci 4:309-356. Medline

Lu HD, Roe AW (2008) Functional organization of color domains in V1 and
V2 of macaque monkey revealed by optical imaging. Cereb Cortex 18: 516-533. CrossRef Medline

Maldonado PE, Gray CM (1996) Heterogeneity in local distributions of orientation-selective neurons in the cat primary visual cortex. Vis Neurosci 13:509-516. CrossRef Medline

Maldonado PE, Gödecke I, Gray CM, Bonhoeffer T (1997) Orientation selectivity in pinwheel centers in cat striate cortex. Science 276:1551-1555. CrossRef Medline

Mariño J, Schummers J, Lyon DC, Schwabe L, Beck O, Wiesing P, Obermayer K, Sur M (2005) Invariant computations in local cortical networks with balanced excitation and inhibition. Nat Neurosci 8:194-201. CrossRef Medline

Nauhaus I, Benucci A, Carandini M, Ringach DL (2008) Neuronal selectivity and local map structure in visual cortex. Neuron 57:673-679. CrossRef Medline

Nauhaus I, Nielsen KJ, Callaway EM (2012a) Nonlinearity of two-photon $\mathrm{Ca}^{2+}$ imaging yields distorted measurements of tuning for $\mathrm{V} 1$ neuronal populations. J Neurophysiol 107:923-936. CrossRef Medline

Nauhaus I, Nielsen KJ, Disney AA, Callaway EM (2012b) Orthogonal micro-organization of orientation and spatial frequency in primate primary visual cortex. Nat Neurosci 15:1683-1690. CrossRef Medline

Nimmerjahn A, Kirchhoff F, Kerr JN, Helmchen F (2004) Sulforhodamine 101 as a specific marker of astroglia in the neocortex in vivo. Nat Methods 1:31-37. CrossRef Medline

Ohki K, Chung S, Ch'ng YH, Kara P, Reid RC (2005) Functional imaging with cellular resolution reveals precise micro-architecture in visual cortex. Nature 433:597-603. CrossRef Medline

Ohki K, Chung S, Kara P, Hübener M, Bonhoeffer T, Reid RC (2006) Highly ordered arrangement of single neurons in orientation pinwheels. Nature 442:925-928. CrossRef Medline

Okamoto T, Ikezoe K, Tamura H, Watanabe M, Aihara K, Fujita I (2011) Predicted contextual modulation varies with distance from pinwheel centers in the orientation preference map. Sci Rep 1:114. Medline

O’Keefe LP, Levitt JB, Kiper DC, Shapley RM, Movshon JA (1998) Functional organization of owl monkey lateral geniculate nucleus and visual cortex. J Neurophysiol 80:594-609. Medline

Polimeni JR, Granquist-Fraser D, Wood RJ, Schwartz EL (2005) Physical limits to spatial resolution of optical recording: clarifying the spatial structure of cortical hypercolumns. Proc Natl Acad Sci U S A 102:4158 4163. CrossRef Medline

Popilskis SJ, Kohn DF (1997) Anesthesia and analgesia in nonhuman primates. In: Anesthesia and analgesia in laboratory animals (Kohn DF, Wixson SK, White WJ, Benson GJ, eds), pp 233-255. San Diego: Academic.

Ringach DL (2007) On the origin of the functional architecture of the cortex. PLoS One 2:e251. CrossRef Medline

Ringach DL, Shapley RM, Hawken MJ (2002) Orientation selectivity in macaque V1: diversity and laminar dependence. J Neurosci 22:5639-5651. Medline

Sato H, Katsuyama N, Tamura H, Hata Y, Tsumoto T (1995) Mechanisms underlying direction selectivity of neurons in the primary visual cortex of the macaque. J Neurophysiol 74:1382-1394. Medline

Sato TR, Gray NW, Mainen ZF, Svoboda K (2007) The functional microarchitecture of the mouse barrel cortex. PLoS Biol 5:e189. CrossRef Medline

Schiller PH, Finlay BL, Volman SF (1976) Quantitative studies of single-cell properties in monkey striate cortex. II. Orientation specificity and ocular dominance. J Neurophysiol 39:1320-1333. Medline

Schummers J, Mariño J, Sur M (2002) Synaptic integration by V1 neurons depends on location within the orientation map. Neuron 36:969-978. CrossRef Medline

Stosiek C, Garaschuk O, Holthoff K, Konnerth A (2003) In vivo two-photon calcium imaging of neuronal networks. Proc Natl Acad Sci U S A 100: 7319-7324. CrossRef Medline

Swindale NV (1998) Orientation tuning curves: empirical description and estimation of parameters. Biol Cybern 78:45-56. CrossRef Medline

Tamura H, Kaneko H, Kawasaki K, Fujita I (2004) Presumed inhibitory neurons in the macaque inferior temporal cortex: visual response properties and functional interactions with adjacent neurons. J Neurophysiol 91:2782-2796. CrossRef Medline

Ts'o DY, Gilbert CD (1988) The organization of chromatic and spatial interactions in the primate striate cortex. J Neurosci 8:1712-1727. Medline 